Acta Crystallographica Section E

Structure Reports

Online

ISSN 1600-5368

\section{Powder study of hydrochlorothiazide form II}

\section{Alastair Florence, ${ }^{\mathrm{a} *}$ Andrea Johnston, ${ }^{\text {a Philippe Fernandes, }}$, Kenneth Shankland, ${ }^{\text {b }}$ \\ Howard N. E. Stevens, ${ }^{a}$ \\ Sigrunn Osmundsen ${ }^{\mathrm{a}}$ and \\ Alexander B. Mullen ${ }^{a}$}

${ }^{a}$ Department of Pharmaceutical Sciences, University of Strathclyde, 27 Taylor Street, Glasgow G4 0NR, Scotland, and ${ }^{b}$ ISIS Facility, Rutherford Appleton Laboratory, Chilton, Didcot, Oxon OX11 0QX, England

Correspondence e-mail:

alastair.florence@strath.ac.uk

\author{
Key indicators \\ Powder X-ray study \\ $T=298 \mathrm{~K}$ \\ Mean $\sigma(\mathrm{C}-\mathrm{C})=0.002 \AA$ \\ $R$ factor $=0.033$ \\ $w R$ factor $=0.038$ \\ Data-to-parameter ratio $=3.85$
}

For details of how these key indicators were automatically derived from the article, see http://journals.iucr.org/e.
The crystal structure of hydrochlorothiazide form II, $\mathrm{C}_{7} \mathrm{H}_{8} \mathrm{ClN}_{3} \mathrm{O}_{4} \mathrm{~S}_{2}$, was solved by simulated annealing from laboratory X-ray powder diffraction data collected at room temperature to $1.76 \AA$ resolution. Subsequent Rietveld refinement yielded an $R_{\mathrm{wp}}$ of 0.0376 to $1.49 \AA$ resolution. The molecules crystallize in the space group $P 2_{1} / c$ with one molecule in the asymmetric unit. The structure is stabilized by three $\mathrm{N}-\mathrm{H} \cdots \mathrm{N}$ and one $\mathrm{N}-\mathrm{H} \cdots \mathrm{O}$ hydrogen-bonded intermolecular interaction.

\section{Comment}

Hydrochlorothiazide (HCT) is a thiazide diuretic which is known to crystallize in at least one non-solvated form (Dupont \& Dideberg, 1972). A polycrystalline sample of a second polymorph of HCT, form II, (I), was produced using a modified precipitation technique in which an acetone solution of HCT was added to distilled water containing hydroxypropylmethylcellulose (grade E5LV, Dow Chemicals, USA) under<smiles>NS(=O)(=O)c1cc2c(cc1Cl)NCNS2(=O)=O</smiles>

agitation. The resulting precipitate was immediately isolated from solution by membrane filtration. The sample was identified as a new form using multisample X-ray powder diffraction analysis (Florence et al., 2003). The sample was

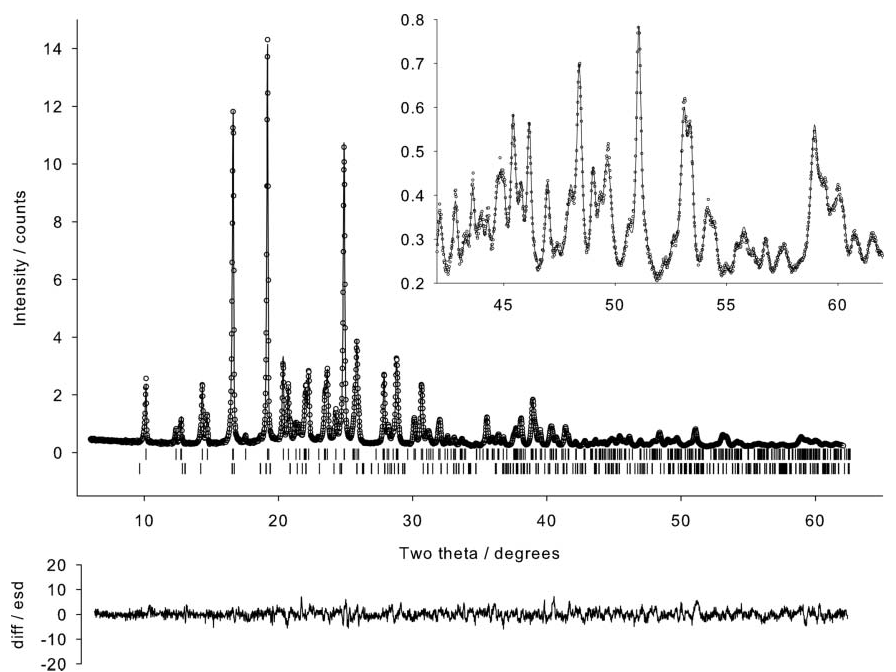

Figure 1

Final observed (points), calculated (line) and difference [( $\left.y_{\text {obs }}-y_{\text {calc }}\right) /$ $\left.\sigma\left(y_{\text {obs }}\right)\right]$ profiles for the Rietveld refinement of the title compound.
Received 14 July 2005 Accepted 25 July 2005 Online 6 August 2005
(C) 2005 International Union of Crystallography Printed in Great Britain - all rights reserved 


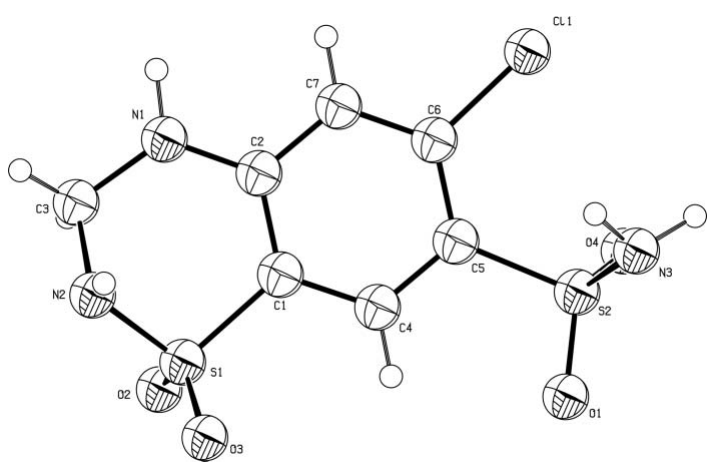

Figure 2

The molecular structure with the atom-numbering scheme. Isotropic displacement spheres are shown at the $50 \%$ probability level.

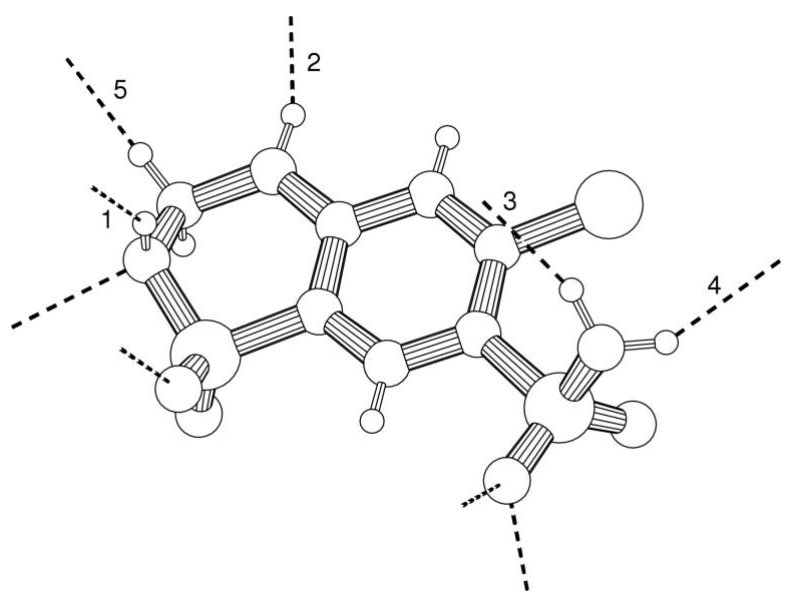

Figure 3

Packing diagram illustrating intermolecular contacts (dashed lines) in the structure of (I). Unique contacts are labelled as follows: $1: \mathrm{N} 2 \cdots \mathrm{O} 3=$ 2.905 (4) $\AA$, O3 in the molecule at $(-x, 1-y,-z) ; 2: \mathrm{N} 1 \cdots \mathrm{O} 1=$ 2.920 (3) $\AA$, O1 in the molecule at $\left(x, 1 / 2-y,-\frac{1}{2}+z\right) ; 3: \mathrm{N} 3 \cdots \mathrm{O} 1=$ 3.121 (3) $\AA$, O1 in the molecule at $\left(1-x, \frac{1}{2}+y, \frac{1}{2}-z\right) ; 4: \mathrm{N} 3 \cdots \mathrm{N} 2=$ 3.214 (2) $\AA$, $\mathrm{N} 2$ in the molecule at $\left(1+x, \frac{1}{2}-y, \frac{1}{2}+z\right) ; 5: \mathrm{C} 3 \cdots \mathrm{O} 2=$ 3.416 (3) $\AA, \mathrm{O} 2$ in the molecule at $\left(-x, \frac{1}{2}+y,-\frac{1}{2}-z\right)$. Contacts calculated and illustrated using PLATON (Spek, 2003; program version 280604).

found to contain a trace amount of HCT form I (Dupont \& Dideberg, 1972).

The crystal structure of (I) was solved by simulated annealing using laboratory X-ray powder diffraction data (Fig. 1). The compound crystallizes in space group $P 2_{1} / c$ with one molecule in the asymmetric unit (Fig. 2). In (I), the N2/S1/ $\mathrm{C} 1 / \mathrm{C} 2 / \mathrm{N} 1 / \mathrm{C} 3$ ring in HCT displays a non-planar conformation, atoms $\mathrm{N} 2$ and $\mathrm{C} 3$ having the largest deviations [0.458 (1) and -0.266 (1) $\AA$, respectively] from the least-squares plane through the aromatic ring. The sulfonamide side chain adopts a torsion angle $\mathrm{N} 3-\mathrm{S} 2-\mathrm{C} 5-\mathrm{C} 6=59.53(19)^{\circ}$, such that $\mathrm{O} 1$ eclipses $\mathrm{H} 4$, and atoms $\mathrm{O} 4$ and $\mathrm{N} 3$ are staggered with respect to Cl1. In HCT form I (Dupont \& Dideberg, 1972), this group is rotated by approximately $120^{\circ}$ compared with (I), such that the amine group lies on the opposite side of the benzothiadiazine ring system.

The crystal structure is stabilized by a series of intermolecular contacts including three $\mathrm{N}-\mathrm{H} \cdots \mathrm{N}$ hydrogen bonds

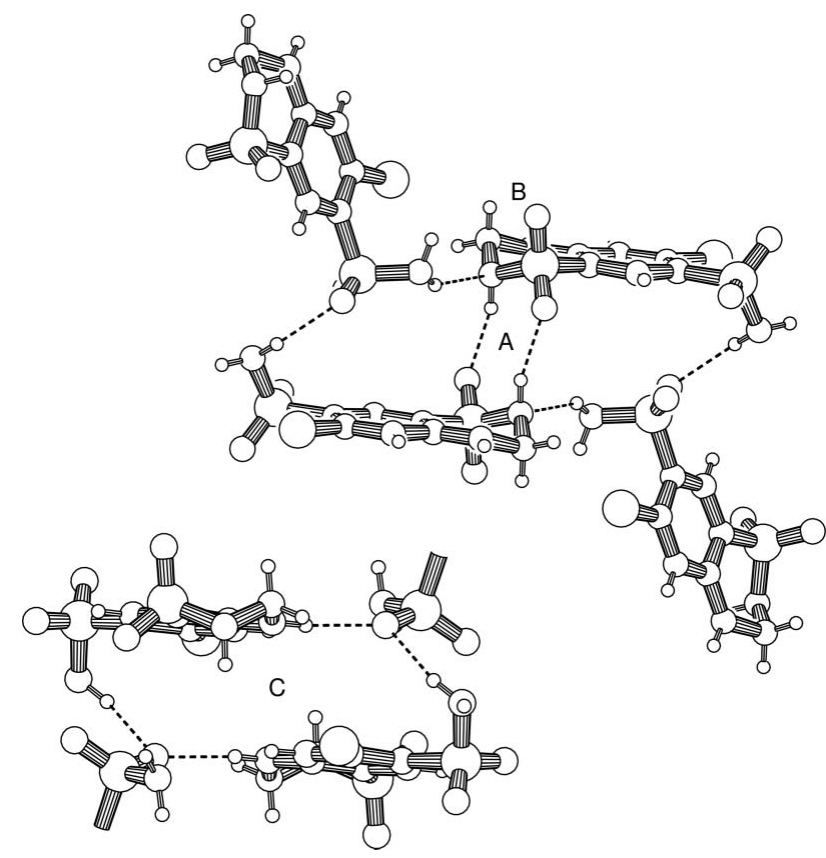

Figure 4

(Top) The $R_{2}^{2}(8)$ (labelled A) and $R_{4}^{4}(24)$ (labelled B) motifs within the structure of (I). (Bottom left) The $R_{4}^{2}(20)$ motif, shown with atoms not involved in the motif omitted for clarity.

(contacts $1-3$, Fig. 3), one $\mathrm{N}-\mathrm{H} \cdots \mathrm{O}$ hydrogen bond (contact 4) and a $\mathrm{C}-\mathrm{H} \cdots \mathrm{O}$ contact (contact 5). Contact 1 forms a centrosymmetric $R_{2}^{2}(8)$ dimer motif (Fig. 4, A), whilst contacts 3 and 4 produce a larger $R_{4}^{4}(24)$ motif (Fig. 4, B) connecting four molecules of HCT. Contacts 2 and 3 also combine to produce an $R_{4}^{2}(20)$ ring motif (Fig. 4, C).

\section{Experimental}

A sample of (I), obtained using the method described in the Comment, was lightly ground in a mortar, loaded into a $0.7 \mathrm{~mm}$ borosilicate glass capillary and mounted on the diffractometer. Data were collected from a sample in a rotating $0.7 \mathrm{~mm}$ borosilicate glass capillary using a variable count time scheme (Shankland et al., 1997; Hill \& Madsen, 2002).

\section{Crystal data}

$\mathrm{C}_{7} \mathrm{H}_{8} \mathrm{ClN}_{3} \mathrm{O}_{4} \mathrm{~S}_{2}$

$M_{r}=297.75$

Monoclinic, $P 2_{b} / c$

$a=9.4884(5) \AA$

$b=8.3334(4) \AA$

$c=15.1309$ (7) $\AA$

$\beta=113.2087(19)^{\circ}$

$V=1099.59(9) \AA^{3}$

$Z=4$

Data collection

Bruker AXS D8 Advance diffractometer

Specimen mounting: $0.7 \mathrm{~mm}$ borosilicate capillary

Specimen mounted in transmission mode

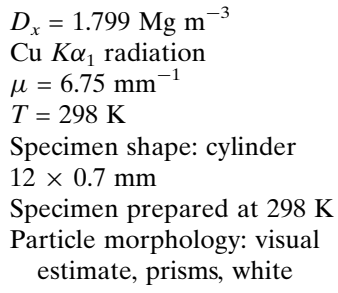

Scan method: step Absorption correction: none $2 \theta_{\min }=5.0,2 \theta_{\max }=65.0^{\circ}$ Increment in $2 \theta=0.017^{\circ}$ 


\section{Refinement}

Refinement on $I_{\text {net }}$

$R_{\mathrm{p}}=0.033$

$R_{\text {wp }}=0.038$

$R_{\text {exp }}=0.023$

$R_{\mathrm{B}}=0.013$

$S=1.64$

Excluded region(s): 62.1 to $65.0 \%$ due to poor signal-to-noise

Profile function: fundamental parameters with axial divergence correction

Table 1

Selected geometric parameters $\left(\AA{ }^{\circ}{ }^{\circ}\right)$.

\begin{tabular}{llll}
\hline $\mathrm{C} 11-\mathrm{C} 6$ & $1.730(2)$ & $\mathrm{S} 2-\mathrm{O} 4$ & $1.429(3)$ \\
$\mathrm{S} 1-\mathrm{O} 2$ & $1.425(3)$ & $\mathrm{S} 2-\mathrm{N} 3$ & $1.633(2)$ \\
$\mathrm{S} 1-\mathrm{O} 3$ & $1.426(2)$ & $\mathrm{S} 2-\mathrm{C} 5$ & $1.772(2)$ \\
$\mathrm{S} 1-\mathrm{N} 2$ & $1.6438(16)$ & $\mathrm{N} 1-\mathrm{C} 2$ & $1.358(2)$ \\
$\mathrm{S} 1-\mathrm{C} 1$ & $1.7650(17)$ & $\mathrm{N} 1-\mathrm{C} 3$ & $1.473(2)$ \\
$\mathrm{S} 2-\mathrm{O} 1$ & $1.428(2)$ & $\mathrm{N} 2-\mathrm{C} 3$ & $1.441(2)$ \\
& & & \\
$\mathrm{O} 2-\mathrm{S} 1-\mathrm{O} 3$ & $119.4(2)$ & $\mathrm{C} 2-\mathrm{N} 1-\mathrm{C} 3$ & $123.27(16)$ \\
$\mathrm{O} 2-\mathrm{S} 1-\mathrm{N} 2$ & $110.42(13)$ & $\mathrm{S} 1-\mathrm{N} 2-\mathrm{C} 3$ & $113.58(11)$ \\
$\mathrm{O} 2-\mathrm{S} 1-\mathrm{C} 1$ & $108.92(17)$ & $\mathrm{S} 1-\mathrm{C} 1-\mathrm{C} 2$ & $119.80(11)$ \\
$\mathrm{O} 3-\mathrm{S} 1-\mathrm{N} 2$ & $106.39(18)$ & $\mathrm{S} 1-\mathrm{C} 1-\mathrm{C} 4$ & $119.55(11)$ \\
$\mathrm{O} 3-\mathrm{S} 1-\mathrm{C} 1$ & $109.25(10)$ & $\mathrm{N} 1-\mathrm{C} 2-\mathrm{C} 1$ & $121.84(13)$ \\
$\mathrm{N} 2-\mathrm{S} 1-\mathrm{C} 1$ & $100.90(10)$ & $\mathrm{N} 1-\mathrm{C} 2-\mathrm{C} 7$ & $120.34(13)$ \\
$\mathrm{O} 1-\mathrm{S} 2-\mathrm{O} 4$ & $122.35(19)$ & $\mathrm{N} 1-\mathrm{C} 3-\mathrm{N} 2$ & $117.40(12)$ \\
$\mathrm{O} 1-\mathrm{S} 2-\mathrm{N} 3$ & $106.27(14)$ & $\mathrm{S} 2-\mathrm{C} 5-\mathrm{C} 4$ & $124.83(13)$ \\
$\mathrm{O} 1-\mathrm{S} 2-\mathrm{C} 5$ & $105.68(13)$ & $\mathrm{S} 2-\mathrm{C} 5-\mathrm{C} 6$ & $121.28(16)$ \\
$\mathrm{O} 4-\mathrm{S} 2-\mathrm{N} 3$ & $107.67(14)$ & $\mathrm{Cl} 1-\mathrm{C} 6-\mathrm{C} 5$ & $117.16(14)$ \\
$\mathrm{O} 4-\mathrm{S} 2-\mathrm{C} 5$ & $108.82(13)$ & $\mathrm{Cl} 1-\mathrm{C} 6-\mathrm{C} 7$ & \\
$\mathrm{~N} 3-\mathrm{S} 2-\mathrm{C} 5$ & $104.75(10)$ & & \\
\hline
\end{tabular}

Table 2

Hydrogen-bond geometry $\left(\AA,^{\circ}\right)$.

\begin{tabular}{lllll}
\hline$D-\mathrm{H} \cdots A$ & $D-\mathrm{H}$ & $\mathrm{H} \cdots A$ & $D \cdots A$ & $D-\mathrm{H} \cdots A$ \\
\hline $\mathrm{N} 2-\mathrm{H} 5 \cdots \mathrm{O} 3^{\mathrm{i}}$ & $0.95(1)$ & $2.03(1)$ & $2.905(4)$ & $152(1)$ \\
$\mathrm{N} 1-\mathrm{H} 6 \cdots \mathrm{O} 1^{\text {ii }}$ & $0.94(1)$ & $2.02(1)$ & $2.920(3)$ & $159(1)$ \\
$\mathrm{N}^{\mathrm{iii}}-\mathrm{H} 7 \cdots \mathrm{O} 1^{\text {ii }}$ & $0.95(1)$ & $2.21(1)$ & $3.121(3)$ & $161(1)$ \\
$\mathrm{N} 3-\mathrm{H} 8 \cdots \mathrm{N} 2^{\text {v }}$ & $0.95(1)$ & $2.37(1)$ & $3.214(2)$ & $149(1)$ \\
$\mathrm{C} 3-\mathrm{H} 1 \cdots \mathrm{O} 2^{\mathrm{v}}$ & $0.95(1)$ & $2.56(1)$ & $3.416(3)$ & $150(1)$ \\
$\mathrm{C} 4-\mathrm{H} 4 \cdots \mathrm{O} 1$ & $0.95(1)$ & $2.37(1)$ & $2.803(3)$ & $108(1)$ \\
\hline
\end{tabular}

Symmetry codes: (i) $-x,-y+1,-z$; (ii) $x,-y+\frac{1}{2},+z-\frac{1}{2}$; (iii) $-x+1,+y+\frac{1}{2},-z+\frac{1}{2}$; (iv) $x+1,-y+\frac{1}{2},+z+\frac{1}{2}$; (v) $-x,+y+\frac{1}{2},-z-\frac{1}{2}$.

The diffraction pattern indexed to a monoclinic cell $[M(20)=25.9$, $F(20)=70.7$; DICVOL-91; Boultif \& Louer, 1991), and space group $P 2_{1} / c$ was assigned from volume considerations and a statistical consideration of the systematic absences (Markvardsen et al., 2001). The data set was background subtracted and truncated to $52.2^{\circ} 2 \theta$ for Pawley (1981) fitting $\left(\chi_{\text {Pawley }}^{2}=5.17\right)$ and the structure was solved using the simulated annealing (SA) global optimization procedure (David et al., 1998), which is now implemented in the DASH computer program (David et al., 1998). The SA structure solution involved the optimization of one molecule of HCT, totaling 7 degrees of freedom. The best SA solution had a favourable $\chi^{2} \mathrm{sA}^{2} \chi_{\text {Pawley }}^{2}$ ratio of 2.6 , a chemically reasonable packing arrange- ment and exhibited no significant misfit to the data. Prior to Rietveld refinement, atoms $\mathrm{H} 7$ and $\mathrm{H} 8$ were set to positions which satisfied the hydrogen bonding contacts within the structure. The solved structure was subsequently refined with data in the range $6.0-62.1^{\circ} 2 \theta$ using a restrained Rietveld (1969) method, as implemented in TOPAS (Coelho, 2003), with the $R_{\mathrm{wp}}$ falling to 0.038 during the refinement. A joint refinement strategy was implemented, in which the structure of HCT form I (Dupont \& Dideberg, 1972) was included to take account of the impurity peaks arising from the presence of a small amount (estimated at less than $5 \%$ ) of this polymorph in the sample. In the course of the refinement, the form I unit-cell and peak-shape parameters were allowed to vary, whilst all atomic coordinates were fixed. All atomic positions (including $\mathrm{H}$ atoms) for the form II structure were refined, subject to a series of restraints on bond lengths, bond angles and planarity. $U_{\text {iso }}(\mathrm{H})$ values were set at $0.044 \AA^{2}$. A spherical harmonics correction of intensities for preferred orientation was applied in the final refinement. The observed and calculated diffraction patterns for the refined crystal structure are shown in Fig. 1.

Data collection: DIFFRAC plus XRD Commander (Kienle \& Jacob, 2003); cell refinement: TOPAS (Coelho, 2003); data reduction: $D A S H$ (David et al., 2001); program(s) used to solve structure: $D A S H$; program(s) used to refine structure: TOPAS; molecular graphics: PLATON (Spek, 2003); software used to prepare material for publication: enCIFer (Allen et al., 2004).

We thank the Basic Technology programme of the UK Research Councils for funding under the project Control and Prediction of the Organic Solid State (http://www.cposs.org.uk). We also thank the CCLRC Centre for Molecular Structure and Dynamics and Pharmaceutics International Inc. (Baltimore, USA) for studentship funding for PF and SO, respectively, and the EPSRC for grant GR/N07462/01.

\section{References}

Allen, F. H., Johnson, O., Shields, G. P., Smith, B. R. \& Towler, M. (2004). J. Appl. Cryst. 37, 335-338.

Boultif, A. \& Louer, D. (1991). J. Appl. Cryst. 24, 987-993.

Coelho, A. A. (2003). TOPAS User Manual. Version 3.1. Bruker AXS GmbH, Karlsruhe, Germany.

David, W. I. F., Shankland, K., Cole, J., Maginn, S., Motherwell, W. D. S. \& Taylor, R. (2001). DASH. Version 3.0 User Manual. Cambridge Crystallographic Data Centre, England.

David, W. I. F., Shankland, K. \& Shankland, N. (1998). Chem. Commun. pp. 931-932.

Dupont, L. \& Dideberg, O. (1972). Acta Cryst. B28, 2340-2347. (In French.)

Florence, A. J., Baumgartner, B., Weston, C., Shankland, N., Kennedy, A. R., Shankland, K. \& David, W. I. F. (2003). J. Pharm. Sci. 92, 1930-1938.

Hill, R. J. \& Madsen, I. C. (2002). Structure Determination from Powder Diffraction Data, edited by W. I. F. David, K. Shankland, L. B. McCusker \& Ch. Baerlocher, pp.114-116. Oxford University Press.

Kienle, M. \& Jacob, M. (2003). DIFFRAC plus XRD Commander. Version 2.3. Bruker AXS GmbH, Karlsruhe, Germany.

Markvardsen, A. J., David, W. I. F., Johnson, J. C. \& Shankland, K. (2001). Acta Cryst. A57, 47-54.

Pawley, G. S. (1981). J. Appl. Cryst. 14, 357-361.

Rietveld, H. M. (1969). J. Appl. Cryst. 2, 65-71.

Shankland, K., David, W. I. F. \& Sivia, D. S. (1997). J. Mater. Chem. 7, 569-572.

Spek, A. L. (2003). J. Appl. Cryst. 36, 7-13. 


\section{supporting information}

Acta Cryst. (2005). E61, o2798-o2800 [https://doi.org/10.1107/S1600536805023640]

Powder study of hydrochlorothiazide form II

Alastair Florence, Andrea Johnston, Philippe Fernandes, Kenneth Shankland, Howard N. E. Stevens, Sigrunn Osmundsen and Alexander B. Mullen

(I)

\section{Crystal data}

$\mathrm{C}_{7} \mathrm{H}_{8} \mathrm{ClN}_{3} \mathrm{O}_{4} \mathrm{~S}_{2}$

$M_{r}=297.75$

Monoclinic, $P 2_{1} / c$

Hall symbol: $-\mathrm{P} 2 \mathrm{ybc}$

$a=9.4884(5) \AA$

$b=8.3334(4) \AA$

$c=15.1309(7) \AA$

$\beta=113.2087(19)^{\circ}$

$V=1099.59(9) \AA^{3}$

$Z=4$

$F(000)=608.0$

$D_{\mathrm{x}}=1.799 \mathrm{Mg} \mathrm{m}^{-3}$

$\mathrm{Cu} K \alpha_{1}$ radiation, $\lambda=1.54056 \AA$

$\mu=6.75 \mathrm{~mm}^{-1}$

$T=298 \mathrm{~K}$

Particle morphology: visual estimate, prisms

white

cylinder, $12 \times 0.7 \mathrm{~mm}$

Specimen preparation: Prepared at $298 \mathrm{~K}$

\section{Data collection}

Bruker AXS D8 Advance diffractometer

Radiation source: sealed X-ray tube, BrukerAXS D8

Primary focussing, Ge 111 monochromator

Refinement

Least-squares matrix: selected elements only

$R_{\mathrm{p}}=0.033$

$R_{\mathrm{wp}}=0.038$

$R_{\text {exp }}=0.023$

$R_{\text {Bragg }}=0.013$

3529 data points

Excluded region(s): 62.1 to 65.0 due to poor signal to noise

Profile function: Fundamental parameters with axial divergence correction

92 parameters

Specimen mounting: $0.7 \mathrm{~mm}$ borosilicate capillary

Data collection mode: transmission

Scan method: step

$2 \theta_{\min }=5.0^{\circ}, 2 \theta_{\max }=65.0^{\circ}, 2 \theta_{\text {step }}=0.017^{\circ}$

47 restraints

1 constraint

Only H-atom coordinates refined

Weighting scheme based on measured s.u.'s $1 / \sigma\left(\mathrm{Y}_{\mathrm{obs}}\right)^{2}$

$(\Delta / \sigma)_{\max }=0.005$

Background function: Chebyshev polynomial

Preferred orientation correction: A spherical harmonics-based preferred orientation correction was applied with Topas during the Rietveld refinement.

Special details

Geometry. Bond distances, angles etc. have been calculated using the rounded fractional coordinates. All su's are estimated from the variances of the (full) variance-covariance matrix. The cell e.s.d.'s are taken into account in the estimation of distances, angles and torsion angles 
Fractional atomic coordinates and isotropic or equivalent isotropic displacement parameters $\left(\AA^{2}\right)$

\begin{tabular}{lllll}
\hline & $x$ & $y$ & $z$ & $U_{\text {iso }} * / U_{\mathrm{eq}}$ \\
\hline C11 & $0.75173(15)$ & $0.1570(3)$ & $0.12511(14)$ & $0.0269(4)^{*}$ \\
S1 & $0.04110(12)$ & $0.25043(13)$ & $-0.05257(7)$ & $0.0269(4)^{*}$ \\
S2 & $0.53828(12)$ & $-0.00769(14)$ & $0.22842(7)$ & $0.0269(4)^{*}$ \\
O1 & $0.41184(17)$ & $-0.0340(4)$ & $0.25537(14)$ & $0.0269(4)^{*}$ \\
O2 & $-0.0243(4)$ & $0.1139(3)$ & $-0.111(2)$ & $0.0269(4)^{*}$ \\
O3 & $-0.0117(2)$ & $0.2956(5)$ & $0.02000(11)$ & $0.0269(4)^{*}$ \\
O4 & $0.6253(3)$ & $-0.1367(3)$ & $0.21308(19)$ & $0.0269(4)^{*}$ \\
N1 & $0.26907(15)$ & $0.3922(3)$ & $-0.12379(11)$ & $0.0269(4)^{*}$ \\
N2 & $0.02352(17)$ & $0.41019(14)$ & $-0.11982(8)$ & $0.0269(4)^{*}$ \\
N3 & $0.65720(17)$ & $0.10948(14)$ & $0.31064(14)$ & $0.0269(4)^{*}$ \\
C1 & $0.24235(12)$ & $0.2284(2)$ & $-0.00040(9)$ & $0.0269(4)^{*}$ \\
C2 & $0.33184(13)$ & $0.3004(2)$ & $-0.04357(9)$ & $0.0269(4)^{*}$ \\
C3 & $0.10325(14)$ & $0.39732(15)$ & $-0.18320(9)$ & $0.0269(4)^{*}$ \\
C4 & $0.30954(13)$ & $0.1390(2)$ & $0.08259(9)$ & $0.0269(4)^{*}$ \\
C5 & $0.46572(15)$ & $0.1121(2)$ & $0.12321(12)$ & $0.0269(4)^{*}$ \\
C6 & $0.55492(15)$ & $0.1828(3)$ & $0.07942(12)$ & $0.0269(4)^{*}$ \\
C7 & $0.49079(13)$ & $0.2748(2)$ & $-0.00157(9)$ & $0.0269(4)^{*}$ \\
H1 & $0.0814(8)$ & $0.4892(11)$ & $-0.2234(7)$ & $0.044^{*}$ \\
H2 & $0.0707(10)$ & $0.3011(11)$ & $-0.2187(6)$ & $0.044^{*}$ \\
H3 & $0.5541(9)$ & $0.3160(10)$ & $-0.0320(6)$ & $0.044^{*}$ \\
H4 & $0.2476(9)$ & $0.0937(11)$ & $0.1121(6)$ & $0.044^{*}$ \\
H5 & $0.0555(9)$ & $0.5041(11)$ & $-0.0813(6)$ & $0.044^{*}$ \\
H6 & $0.3375(9)$ & $0.4321(11)$ & $-0.1498(6)$ & $0.044^{*}$ \\
H7 & $0.6580(8)$ & $0.2158(10)$ & $0.2881(6)$ & $0.044^{*}$ \\
H8 & $0.7567(9)$ & $0.0647(11)$ & $0.3389(6)$ & $0.044^{*}$ \\
& & & & \\
\hline
\end{tabular}

Geometric parameters $\left(\AA,{ }^{\circ}\right)$

\begin{tabular}{llll}
\hline $\mathrm{C} 11-\mathrm{C} 6$ & $1.730(2)$ & $\mathrm{N} 2-\mathrm{H} 5$ & $0.951(9)$ \\
$\mathrm{S} 1-\mathrm{O} 2$ & $1.425(3)$ & $\mathrm{N} 3-\mathrm{H} 7$ & $0.951(8)$ \\
$\mathrm{S} 1-\mathrm{O} 3$ & $1.426(2)$ & $\mathrm{N} 3-\mathrm{H} 8$ & $0.946(9)$ \\
$\mathrm{S} 1-\mathrm{N} 2$ & $1.6438(16)$ & $\mathrm{C} 1-\mathrm{C} 2$ & $1.3946(19)$ \\
$\mathrm{S} 1-\mathrm{C} 1$ & $1.7650(17)$ & $\mathrm{C} 1-\mathrm{C} 4$ & $1.381(2)$ \\
$\mathrm{S} 2-\mathrm{O} 1$ & $1.428(2)$ & $\mathrm{C} 2-\mathrm{C} 7$ & $1.4031(19)$ \\
$\mathrm{S} 2-\mathrm{O} 4$ & $1.429(3)$ & $\mathrm{C} 4-\mathrm{C} 5$ & $1.381(2)$ \\
$\mathrm{S} 2-\mathrm{N} 3$ & $1.633(2)$ & $\mathrm{C} 5-\mathrm{C} 6$ & $1.394(2)$ \\
$\mathrm{S} 2-\mathrm{C} 5$ & $1.772(2)$ & $\mathrm{C} 6-\mathrm{C} 7$ & $1.368(2)$ \\
$\mathrm{N} 1-\mathrm{C} 2$ & $1.358(2)$ & $\mathrm{C} 3-\mathrm{H} 1$ & $0.949(9)$ \\
$\mathrm{N} 1-\mathrm{C} 3$ & $1.473(2)$ & $\mathrm{C} 3-\mathrm{H} 2$ & $0.947(9)$ \\
$\mathrm{N} 2-\mathrm{C} 3$ & $1.441(2)$ & $\mathrm{C} 4-\mathrm{H} 4$ & $0.946(9)$ \\
$\mathrm{N} 1-\mathrm{H} 6$ & $0.943(9)$ & $\mathrm{C} 7-\mathrm{H} 3$ & $119.55(11)$ \\
& & & $117.82(13)$ \\
$\mathrm{O} 2-\mathrm{S} 1-\mathrm{O} 3$ & $119.4(2)$ & $\mathrm{S} 1-\mathrm{C} 1-\mathrm{C} 4$ & $121.84(13)$ \\
$\mathrm{O} 2-\mathrm{S} 1-\mathrm{N} 2$ & $110.42(13)$ & $\mathrm{C} 1-\mathrm{C} 2-\mathrm{C} 7$ & \\
$\mathrm{O} 2-\mathrm{S} 1-\mathrm{C} 1$ & $108.92(17)$ & $\mathrm{N} 1-\mathrm{C} 2-\mathrm{C} 1$ &
\end{tabular}




\begin{tabular}{|c|c|c|c|}
\hline $\mathrm{O} 3-\mathrm{S} 1-\mathrm{N} 2$ & $106.39(18)$ & $\mathrm{N} 1-\mathrm{C} 2-\mathrm{C} 7$ & $120.34(13)$ \\
\hline $\mathrm{O} 3-\mathrm{S} 1-\mathrm{C} 1$ & $109.25(10)$ & $\mathrm{N} 1-\mathrm{C} 3-\mathrm{N} 2$ & $108.10(11)$ \\
\hline $\mathrm{N} 2-\mathrm{S} 1-\mathrm{C} 1$ & $100.90(10)$ & $\mathrm{C} 1-\mathrm{C} 4-\mathrm{C} 5$ & $121.48(13)$ \\
\hline $\mathrm{O} 1-\mathrm{S} 2-\mathrm{O} 4$ & $122.35(19)$ & $\mathrm{S} 2-\mathrm{C} 5-\mathrm{C} 4$ & $117.40(12)$ \\
\hline $\mathrm{O} 1-\mathrm{S} 2-\mathrm{N} 3$ & $106.27(14)$ & $\mathrm{S} 2-\mathrm{C} 5-\mathrm{C} 6$ & $124.83(13)$ \\
\hline $\mathrm{O} 1-\mathrm{S} 2-\mathrm{C} 5$ & $105.68(13)$ & $\mathrm{C} 4-\mathrm{C} 5-\mathrm{C} 6$ & $117.77(15)$ \\
\hline $\mathrm{O} 4-\mathrm{S} 2-\mathrm{N} 3$ & $107.67(14)$ & $\mathrm{Cl} 1-\mathrm{C} 6-\mathrm{C} 5$ & $121.28(16)$ \\
\hline $\mathrm{O} 4-\mathrm{S} 2-\mathrm{C} 5$ & $108.82(13)$ & $\mathrm{Cl} 1-\mathrm{C} 6-\mathrm{C} 7$ & $117.16(14)$ \\
\hline $\mathrm{N} 3-\mathrm{S} 2-\mathrm{C} 5$ & $104.75(10)$ & $\mathrm{C} 5-\mathrm{C} 6-\mathrm{C} 7$ & $121.56(15)$ \\
\hline $\mathrm{C} 2-\mathrm{N} 1-\mathrm{C} 3$ & $123.27(16)$ & $\mathrm{C} 2-\mathrm{C} 7-\mathrm{C} 6$ & $120.67(13)$ \\
\hline $\mathrm{S} 1-\mathrm{N} 2-\mathrm{C} 3$ & $113.58(11)$ & $\mathrm{N} 1-\mathrm{C} 3-\mathrm{H} 1$ & $109.0(6)$ \\
\hline $\mathrm{C} 2-\mathrm{N} 1-\mathrm{H} 6$ & $116.1(6)$ & $\mathrm{N} 1-\mathrm{C} 3-\mathrm{H} 2$ & $110.7(6)$ \\
\hline $\mathrm{C} 3-\mathrm{N} 1-\mathrm{H} 6$ & $118.7(5)$ & $\mathrm{N} 2-\mathrm{C} 3-\mathrm{H} 1$ & $109.1(6)$ \\
\hline $\mathrm{S} 1-\mathrm{N} 2-\mathrm{H} 5$ & $110.9(5)$ & $\mathrm{N} 2-\mathrm{C} 3-\mathrm{H} 2$ & $107.5(6)$ \\
\hline $\mathrm{C} 3-\mathrm{N} 2-\mathrm{H} 5$ & $110.2(6)$ & $\mathrm{H} 1-\mathrm{C} 3-\mathrm{H} 2$ & $112.3(8)$ \\
\hline $\mathrm{S} 2-\mathrm{N} 3-\mathrm{H} 7$ & $112.6(5)$ & $\mathrm{C} 1-\mathrm{C} 4-\mathrm{H} 4$ & $119.6(5)$ \\
\hline $\mathrm{S} 2-\mathrm{N} 3-\mathrm{H} 8$ & $112.0(6)$ & $\mathrm{C} 5-\mathrm{C} 4-\mathrm{H} 4$ & $118.9(6)$ \\
\hline $\mathrm{H} 7-\mathrm{N} 3-\mathrm{H} 8$ & $112.5(8)$ & $\mathrm{C} 2-\mathrm{C} 7-\mathrm{H} 3$ & $119.7(5)$ \\
\hline $\mathrm{C} 2-\mathrm{C} 1-\mathrm{C} 4$ & $120.66(12)$ & $\mathrm{C} 6-\mathrm{C} 7-\mathrm{H} 3$ & $119.5(5)$ \\
\hline $\mathrm{S} 1-\mathrm{C} 1-\mathrm{C} 2$ & $119.80(11)$ & & \\
\hline $\mathrm{O} 2-\mathrm{S} 1-\mathrm{N} 2-\mathrm{C} 3$ & $-62.4(2)$ & $\mathrm{S} 1-\mathrm{N} 2-\mathrm{C} 3-\mathrm{N} 1$ & $-66.39(16)$ \\
\hline $\mathrm{O} 3-\mathrm{S} 1-\mathrm{N} 2-\mathrm{C} 3$ & $166.72(13)$ & $\mathrm{C} 2-\mathrm{C} 1-\mathrm{C} 4-\mathrm{C} 5$ & $-2.8(2)$ \\
\hline $\mathrm{C} 1-\mathrm{S} 1-\mathrm{N} 2-\mathrm{C} 3$ & $52.73(12)$ & $\mathrm{S} 1-\mathrm{C} 1-\mathrm{C} 2-\mathrm{C} 7$ & $-177.71(12)$ \\
\hline $\mathrm{O} 2-\mathrm{S} 1-\mathrm{C} 1-\mathrm{C} 2$ & $96.13(19)$ & $\mathrm{C} 4-\mathrm{C} 1-\mathrm{C} 2-\mathrm{N} 1$ & $-178.32(17)$ \\
\hline $\mathrm{O} 2-\mathrm{S} 1-\mathrm{C} 1-\mathrm{C} 4$ & $-83.47(19)$ & $\mathrm{S} 1-\mathrm{C} 1-\mathrm{C} 2-\mathrm{N} 1$ & $2.1(2)$ \\
\hline $\mathrm{O} 3-\mathrm{S} 1-\mathrm{C} 1-\mathrm{C} 2$ & $-131.9(2)$ & $\mathrm{S} 1-\mathrm{C} 1-\mathrm{C} 4-\mathrm{C} 5$ & $176.79(13)$ \\
\hline $\mathrm{O} 3-\mathrm{S} 1-\mathrm{C} 1-\mathrm{C} 4$ & $48.5(2)$ & $\mathrm{C} 4-\mathrm{C} 1-\mathrm{C} 2-\mathrm{C} 7$ & $1.9(2)$ \\
\hline $\mathrm{N} 2-\mathrm{S} 1-\mathrm{C} 1-\mathrm{C} 2$ & $-20.07(15)$ & $\mathrm{N} 1-\mathrm{C} 2-\mathrm{C} 7-\mathrm{C} 6$ & $179.82(19)$ \\
\hline $\mathrm{N} 2-\mathrm{S} 1-\mathrm{C} 1-\mathrm{C} 4$ & $160.33(13)$ & $\mathrm{C} 1-\mathrm{C} 2-\mathrm{C} 7-\mathrm{C} 6$ & $-0.4(2)$ \\
\hline $\mathrm{O} 1-\mathrm{S} 2-\mathrm{C} 5-\mathrm{C} 4$ & $-7.5(2)$ & $\mathrm{C} 1-\mathrm{C} 4-\mathrm{C} 5-\mathrm{S} 2$ & $-178.81(13)$ \\
\hline $\mathrm{O} 1-\mathrm{S} 2-\mathrm{C} 5-\mathrm{C} 6$ & $171.5(2)$ & $\mathrm{C} 1-\mathrm{C} 4-\mathrm{C} 5-\mathrm{C} 6$ & $2.1(3)$ \\
\hline $\mathrm{O} 4-\mathrm{S} 2-\mathrm{C} 5-\mathrm{C} 4$ & $125.57(18)$ & $\mathrm{S} 2-\mathrm{C} 5-\mathrm{C} 6-\mathrm{C} 7$ & $-179.59(15)$ \\
\hline $\mathrm{O} 4-\mathrm{S} 2-\mathrm{C} 5-\mathrm{C} 6$ & $-55.4(2)$ & $\mathrm{S} 2-\mathrm{C} 5-\mathrm{C} 6-\mathrm{Cl} 1$ & $0.7(3)$ \\
\hline $\mathrm{N} 3-\mathrm{S} 2-\mathrm{C} 5-\mathrm{C} 4$ & $-119.51(15)$ & $\mathrm{C} 4-\mathrm{C} 5-\mathrm{C} 6-\mathrm{Cl} 1$ & $179.73(17)$ \\
\hline $\mathrm{N} 3-\mathrm{S} 2-\mathrm{C} 5-\mathrm{C} 6$ & $59.5(2)$ & $\mathrm{C} 4-\mathrm{C} 5-\mathrm{C} 6-\mathrm{C} 7$ & $-0.6(3)$ \\
\hline $\mathrm{C} 3-\mathrm{N} 1-\mathrm{C} 2-\mathrm{C} 7$ & $166.79(16)$ & $\mathrm{C} 5-\mathrm{C} 6-\mathrm{C} 7-\mathrm{C} 2$ & $-0.3(3)$ \\
\hline $\mathrm{C} 3-\mathrm{N} 1-\mathrm{C} 2-\mathrm{C} 1$ & $-13.0(3)$ & $\mathrm{C} 11-\mathrm{C} 6-\mathrm{C} 7-\mathrm{C} 2$ & $179.46(16)$ \\
\hline $\mathrm{C} 2-\mathrm{N} 1-\mathrm{C} 3-\mathrm{N} 2$ & $44.9(2)$ & & \\
\hline
\end{tabular}

Hydrogen-bond geometry $\left(\AA,{ }^{\circ}\right)$

\begin{tabular}{lllll}
\hline$D-\mathrm{H} \cdots A$ & $D-\mathrm{H}$ & $\mathrm{H} \cdots A$ & $D \cdots A$ & $D-\mathrm{H} \cdots A$ \\
\hline $\mathrm{N} 2-\mathrm{H} 5 \cdots \mathrm{O} 3^{\mathrm{i}}$ & $0.95(1)$ & $2.03(1)$ & $2.905(4)$ & $152(1)$ \\
$\mathrm{N} 1-\mathrm{H} 6 \cdots 1^{\mathrm{ii}}$ & $0.94(1)$ & $2.02(1)$ & $2.920(3)$ & $159(1)$ \\
$\mathrm{N} 3-\mathrm{H} 7 \cdots{ }^{i i i}$ & $0.95(1)$ & $2.21(1)$ & $3.121(3)$ & $161(1)$ \\
$\mathrm{N} 3-\mathrm{H} 8 \cdots \mathrm{N} 2^{\text {iv }}$ & $0.95(1)$ & $2.37(1)$ & $3.214(2)$ & $149(1)$
\end{tabular}


supporting information

$\begin{array}{llllr}\mathrm{C} 3-\mathrm{H} 1 \cdots \mathrm{O} 2^{\mathrm{v}} & 0.95(1) & 2.56(1) & 3.416(3) & 150(1) \\ \mathrm{C} 4-\mathrm{H} 4 \cdots \mathrm{O} 1 & 0.95(1) & 2.37(1) & 2.803(3) & 108(1)\end{array}$

Symmetry codes: (i) $-x,-y+1,-z$; (ii) $x,-y+1 / 2, z-1 / 2$; (iii) $-x+1, y+1 / 2,-z+1 / 2$; (iv) $x+1,-y+1 / 2, z+1 / 2$; (v) $-x, y+1 / 2,-z-1 / 2$. 